\title{
ON THE RELATION BETWEEN OPEN AND CLOSED TOPOLOGICAL STRINGS
}

\author{
ANTON KAPUSTIN AND LEV ROZANSKY
}

\begin{abstract}
We discuss the relation between open and closed string correlators using topological string theories as a toy model. We propose that one can reconstruct closed string correlators from the open ones by considering the Hochschild cohomology of the category of D-branes. We compute the Hochschild cohomology of the category of D-branes in topological Landau-Ginzburg models and partially verify the conjecture in this case.
\end{abstract}

\section{InTRODUCTION AND SUMmary}

There exist different viewpoints on the question whether closed or open strings are more fundamental. The more popular viewpoint is that closed strings are simpler (because one does not have to make a choice of a boundary condition) and therefore more fundamental. In this view, the central problem is to classify D-branes and construct open-closed string correlators for a given closed string theory. In practice, complete classification is possible only in very simple (mostly topological) examples. The opposite viewpoint is supported by the observation that closed String Field Theory is vastly more complicated than the open one. While in the open-string case the classical action is cubic [41, in the closed-string case it is non-polynomial [37, 26]. To write down the open SFT action, one has to specify an associative product on the space of states, a differential (i.e. a BRST operator), and an invariant scalar product. Deformations of the closed-string background change these data, so there is a map from the space of closed-string states to the space of infinitesimal deformations of the open string theory.

One difficulty with the first viewpoint is that it is not clear if the spectrum and properties of D-branes are uniquely determined by the closed string theory. In other words, there may exist D-branes which are perfectly consistent by themselves, but mutually incompatible, in the sense that it is not possible to define states which correspond to open strings stretched between two different branes. If one regards a Dbrane as a boundary condition for a string worldsheet, such a situation may appear absurd, but one must remember that up to now there does 
not exist a precise definition of the notion of a "boundary condition" at the quantum level. Instead, one characterizes D-branes as solutions of a complicated set of conditions (see Refs. 27, 18]), the most nontrivial of which is the so-called Cardy condition. With such an axiomatic definition of a D-brane, the situation described above is not ruled out.

If one adopts the second viewpoint, then the central problem is to construct closed string correlators from the open ones. As mentioned above, closed string states are related to infinitesimal deformations of the open-string theory. Suppose for simplicity that there is only a single D-brane in the theory, so that all the information is encoded in an associative algebra $A$ equipped with a BRST differential $Q$ of ghost number one and an invariant scalar product. The pair $(A, Q)$ is called a differential graded algebra (DG-algebra) by mathematicians. Equivalence classes of deformations of these data are described by a certain cohomology theory (Hochschild cohomology of $(A, Q)$ with coefficients in itself). The simplest conjecture is that the space of physical closedstring states is isomorphic to the Hochschild cohomology of $(A, Q)$.

At this point we must be more specific about the kind of string theory we are talking about. In this paper we will only discuss topological string theories. Let us recall how they are constructed. The starting point is a unitary $N=2 d=2$ field theory which can be twisted to a topological field theory. To get a string theory, one has to couple it to topological gravity. On the level of the space of states, this operation is very simple [42, 15]: each state of the TFT gives rise to an infinite sequence of states of increasing ghost number. The first state in this sequence is called a gravitational primary, and the rest are called gravitational descendants. Tree level correlators of primaries can be computed in the TFT, i.e. the coupling to topological gravity plays no role for these correlators. In this paper we will only discuss such correlators, and therefore topological gravity will play no role. The precise conjecture we are making is that for topological strings the spectrum of gravitational primaries is given by the Hochschild cohomology of the category of topological D-branes.

This conjecture is very appealing, because many structures of the open-closed string theory are then automatic. For example, the Hochschild cohomology of any DG-algebra is itself a supercommutative algebra, which may be identified with the algebra of observables in the closed-string TFT. It also has a Lie-type bracket of degree -1, in agreement with the findings of Refs. [46, 45, 30, 14, 35, 31]. Further, consider the cohomology of $Q$, which may be regarded as the space of physical open-string states. It turns out that this space has a natural structure of a module over the Hochschild cohomology of $(A, Q)$, and this allows 
one to define the bulk-boundary map. This will be discussed in more detail below. Many axioms of open-closed TFT (see Ref. [27]) are then easily verified. ${ }^{1}$

The assumption that there is only one D-brane is unrealistic. For example, given a D-brane $M_{0}$, one may consider direct sums of several copies of $M_{0}$, as well as more complicated bound states. If there are many possible D-branes, then one has to take into account open strings with different boundary conditions on the two ends. It is convenient to think of a D-brane as an object of an additive (in fact $\mathbb{C}$-linear) category, and of the space of open strings between two D-branes as the space of morphisms. Then the algebra of open string states for a particular D-brane is its endomorphism algebra. BRST operators give rise to differentials on all spaces of morphisms, so one is actually dealing with a differential graded category (DG category). The grading is given by the ghost number. To get the space of physical open-string states between any two D-branes, one has to compute the cohomology of the BRST operator on the space of morphisms. There is a notion of Hochschild cohomology which classifies equivalence classes of deformations of such categories. Essentially, one lumps together all the objects in the category into a single "total object" and considers the Hochschild cohomology of its endomorphism algebra. This is equivalent to thinking about a $\mathbb{C}$-linear additive category as an "algebra with many objects." It is tempting to conjecture that the space of physical closed strings is isomorphic to the Hochschild cohomology of the category of D-branes. A heuristic argument for this is explained in the end of Section 2. Again, the space of physical open-string states between any two D-branes is naturally a module over the Hochschild cohomology, so the bulk-boundary maps are automatic.

Even when there are many possible D-branes, one can often find a Dbrane $M_{0}$ such that all other D-branes can be obtained as bound states of several copies of $M_{0}$ and its anti-brane. In mathematical terms, this means that the category of D-branes is equivalent to the category of modules of some kind over the endomorphism algebra (which is actually a DG-algebra) of $M_{0}$. Then the Hochschild cohomology of the category of D-branes coincides with the Hochschild cohomology of the endomorphism algebra of $M_{0}$. In the physical case, the role of $M_{0}$ can be played by a space-filling brane.

\footnotetext{
${ }^{1}$ The Cardy condition is an exception in this regard. It seems to be a generalization of the Hirzebruch-Riemann-Roch theorem, and its validity is not at all obvious.
} 
One example where this prescription for reconstructing closed strings is known to work is the topological B-model of a Calabi-Yau manifold $X$. The algebra of closed string states is given by

$$
\oplus_{p, q} H^{p}\left(\Lambda^{q} T X\right) \text {. }
$$

The category of D-branes is believed to be equivalent to the bounded derived category of $X$ denoted $D^{b}(X)$. More precisely, it is a DGcategory whose derived category is believed to be equivalent to $D^{b}(X)$. The appropriate cohomology to compute is the Hochschild cohomology of the sheaf of algebras $\varnothing_{X}$, and one can show that the latter is isomorphic to Eq. (11) [38. This is discussed in more detail in the next section.

In this paper we study another class of topological string theories: topological Landau-Ginzburg models. The closed string sector has been described by C. Vafa [39]. A simple description of the category of Dbranes in LG models has been proposed by M. Kontsevich and derived from physical considerations in Refs. [21, 22, 5] (see also Ref. [28]). It turns out that the category of D-branes can be thought of as the category of CDG-modules over a certain commutative CDG-algebra, where CDG stands for "curved differential graded." (This notion is explained in detail in Ref. [22] and will be recalled below.) For LG models on smooth spaces many correlators have been computed [22]. In this paper we compute the Hochschild cohomology of the category of Landau-Ginzburg branes and show that in this way we can recover the closed string space of states, together with its algebra structure and scalar product, as described in Ref. 39, as well as some open-closed correlators, as described in Ref. 22 .

One may also consider LG models on orbifolds. Such models are particularly important because they provide an alternative description of certain Calabi-Yau sigma-models (these are so-called Gepner models [1]). The closed-string spectrum for LG orbifolds has been described by K. Intriligator and C. Vafa 20, but its interpretation in mathematical terms has been lacking. We check in several examples that the Hochschild cohomology of the category of D-branes on LG orbifolds reproduces the results of Ref. 20] and others. Our examples include the Gepner models for Fermat-type hypersurfaces in projective spaces. A nice feature of the Hochschild cohomology approach is that the 3-point closed-string correlators (i.e. the Yukawa couplings) and the bulk-boundary maps come out automatically.

These results provide evidence that the conjectural identification of the closed string sector with the Hochschild cohomology of the category of D-branes is correct, at least for topological strings, and that 
certain simple open-closed correlators can be computed using the algebraic structure of the Hochschild complex. Hopefully, multi-point and higher-genus correlators can also be extracted from the Hochschild complex. The technique based on the Hochschild complex could be useful for the computation of the space-time superpotential for superstring compactifications with D-branes based on Gepner models [1]. These open-closed superstring backgrounds have been studied in many papers, see e.g. Refs. 36, 16, 4, 17, 6, 1, 19].

\section{Definitions of the Hochschild cohomology}

We begin by recalling various definitions of the Hochschild cohomology of algebras, DG-algebras, and affine and projective varieties. We will pick the most convenient definition and in the next section generalize it to the case of CDG algebras relevant for the Landau-Ginzburg models.

Let $A$ be an associative algebra over $\mathbb{C}$. The Hochschild cochain complex (with coefficients in $A$ ) is the sequence of vector spaces

$$
C^{n}(A)=\operatorname{Hom}_{\mathbb{C}}\left(A^{\otimes n}, A\right), \quad n=0,1, \ldots,
$$

equipped with a differential $\delta: C^{n}(A) \rightarrow C^{n+1}(A)$ defined by the equation

$$
\begin{aligned}
& (\delta f)\left(a_{1}, \ldots, a_{n+1}\right)=a_{1} f\left(a_{2}, \ldots, a_{n}\right) \\
& +\sum_{i=1}^{n}(-1)^{i} f\left(a_{1}, \ldots, a_{i-1}, a_{i} a_{i+1}, a_{i+2}, \ldots, a_{n}\right) \\
& +(-1)^{n+1} f\left(a_{1}, \ldots, a_{n}\right) a_{n+1} .
\end{aligned}
$$

The cohomology of $\delta$ in degree $n$ will be denoted $\operatorname{HH}^{n}(A)$ and called the Hochschild cohomology of $A$ (with coefficients in $A$ ). The more standard notation for it is $\operatorname{HH}^{n}(A, A)$. Each 2-cocycle $f\left(a_{1}, a_{2}\right)$ defines an infinitesimal deformation of the associative product on $A$. That is, if we define a new product by

$$
a \star b=a b+t f(a, b), \quad t \in \mathbb{C},
$$

it will be associative to linear order in $t$ if and only if $\delta f=0$. Trivial infinitesimal deformations (i.e. infinitesimal deformations which lead to an isomorphic algebra) are classified by 2-coboundaries, i.e. 2-cocycles of the form $\delta g$ for some 1-cochain $g(a)$. Thus $\mathrm{HH}^{2}(A)$ classifies nontrivial deformations of the associative algebra structure on $A$. One can give a similar interpretation to the total Hochschild cohomology $\mathrm{HH}^{*}(A)$ : it classifies infinitesimal deformations of $A$ in the class of $A_{\infty}$ algebras, associative algebras being a very special case of $A_{\infty}$ algebras [34. 
If $A$ is a $\mathbb{Z}$-graded or $\mathbb{Z}_{2}$-graded algebra, the Hochschild complex is defined somewhat differently. Let $A_{p}$ be the degree- $p$ component of $A$, so that $A_{p} \cdot A_{q} \subset A_{p+q}$. An element $f$ of $C^{n}(A)$ is said to have an internal degree $p$ if

$$
f\left(a_{1}, \ldots, a_{n}\right) \in A_{p+k_{1}+\cdots+k_{n}}
$$

whenever $a_{i} \in A_{k_{i}}$. Thus each vector space $C^{n}(A)$ is graded by the internal degree, and we define the total degree of an element of $C^{*}(A)$ to be the sum of $n$ and the internal degree. The Hochschild complex is graded by the total degree, and the differential is given by

$$
\begin{aligned}
& (\delta f)\left(a_{1}, \ldots, a_{n+1}\right)=(-1)^{a \cdot f} a_{1} f\left(a_{2}, \ldots, a_{n}\right) \\
& +\sum_{i=1}^{n}(-1)^{i} f\left(a_{1}, \ldots, a_{i-1}, a_{i} a_{i+1}, a_{i+2}, \ldots, a_{n}\right) \\
& \quad+(-1)^{n+1} f\left(a_{1}, \ldots, a_{n}\right) a_{n+1} .
\end{aligned}
$$

Here and below whenever a symbol occurs in the exponential of $(-1)$, it is understood as its internal degree.

Similarly, let $\mathcal{A}=(A, Q)$ be a DG-algebra. The differential $Q$ is a degree-1 derivation $Q: A_{p} \rightarrow A_{p+1}$ which satisfies $Q^{2}=0$. Using $Q$, we can make $C^{n}(A)$ into a complex: one lets

$$
\begin{aligned}
& (Q f)\left(a_{1}, \ldots, a_{n}\right)=Q\left(f\left(a_{1}, \ldots, a_{n}\right)\right) \\
& \quad-\sum_{i=1}^{n}(-1)^{v_{1}+\ldots+v_{i-1}+f+n-1} f\left(a_{1}, \ldots, a_{i-1}, Q a_{i}, a_{i+1}, \ldots, a_{n}\right) .
\end{aligned}
$$

Thus on the bigraded vector space $C^{*}(A)$ we have two differentials: $Q$, which has internal degree 1 and $n$-degree 0 , and $\delta$, which has internal degree 0 and $n$-degree 1 . The total degree for both differentials is 1 , and it is easy to check that $Q$ and $\delta$ commute. The Hochschild cohomology of $\mathcal{A}$ is defined to be the cohomology of $(-1)^{n} Q+\delta$. The vector space $\mathrm{HH}^{2}(\mathcal{A})$ classifies infinitesimal deformations of $(A, Q)$ in the category of DG-algebras, up to quasi-isomorphism. More generally, $\mathrm{HH}^{*}(\mathcal{A})$ classifies deformations of $(A, Q)$ regarded as an $A_{\infty}$ algebra.

Since Hochschild cochains are functions taking value in an algebra, the Hochschild complex has an obvious algebra structure as well (given by the multiplication of functions). The corresponding binary product is called the cup product. The Hochschild coboundary operator $\delta$ is a derivation of the cup product, and therefore the cup product descends to Hochschild cohomology, making it into a $\mathbb{Z}$-graded algebra. It was noted for the first time by M. Gerstenhaber [12] that the latter algebra 
is always supercommutative, even if $A$ is noncommutative. Gerstenhaber also discovered that there is a natural graded Lie bracket on $\mathrm{HH}^{*}(A)$ of degree -1 . The algebraic structure of $\mathrm{HH}^{*}(A)$ is formally the same as that of the space of functions on a $\mathbb{Z}$-graded supermanifold equipped with a Poisson bracket of degree -1 .

There is an equivalent definition of the Hochschild cohomology of an algebra which has a nice geometric interpretation. It is this definition that we will generalize in the next section. Suppose $A$ is commutative; then one can regard $A$ as the algebra of functions on an affine scheme $X=\operatorname{Spec}(A)$. Consider further $A \otimes A$, its spectrum, $\operatorname{Spec}(A \otimes A)=$ $X \times X$, and the diagonal $\Delta \subset X \times X$. One can think of $\Delta$ as a B-brane (read: object of the bounded derived category) on $X \times X$, and consider its open-string spectrum (read: endomorphism algebra). It turns out that the resulting algebra of physical open-string states is precisely the Hochschild cohomology of $A$.

An algebraic version of this definition is also very concise. One can think of $A$ as a left-right bimodule over $A$, or equivalently as a module over $A \otimes A$ (here we still assume that $A$ is commutative). Then the Hochschild cohomology of $A$ is defined as

$$
\mathrm{HH}^{*}(A)=\operatorname{Ext}_{A \otimes A}(A, A) ;
$$

that is, it is the endomorphism algebra of $A$ regarded as an object of the derived category of modules over $A \otimes A$. If $A$ is noncommutative, then $A$ is not a module over $A \otimes A$, but it is a module over $A \otimes A^{o p}$, where $A^{o p}$ is the opposite algebra of $A$. It turns out that in this more general case we have:

$$
\mathrm{HH}^{*}(A)=\operatorname{Ext}_{A \otimes A^{\text {op }}}(A, A) .
$$

To see how this comes about, we need to compute the endomorphisms of $\Delta$ in $D^{b}(X \times X)$. That is, one has to take a projective resolution of $A$ regarded as a module over $A \otimes A^{o p}$, apply to it the operation $\operatorname{Hom}_{A \otimes A^{o p}}(-, A)$, and evaluate the cohomology of the resulting complex of vector spaces. The key point is that for any algebra $A$ with a unit there is a canonical resolution of $A$ by free $A \otimes A^{o p}$ modules:

$$
\cdots \longrightarrow A^{\otimes 4} \longrightarrow A^{\otimes 3} \longrightarrow A^{\otimes 2} .
$$

Each term in this complex is a bimodule over $A$, which is the same as a module over $A \otimes A^{o p}$. If we use this resolution to compute $\operatorname{Ext}^{*}(A, A)$, we get the Hochschild complex.

If we turn our attention to projective or quasi-projective varieties (or schemes) and their derived categories of coherent sheaves, several definitions of the Hochschild cohomology are possible. One possibility is to 
sheafify the Hochschild complex and take the hypercohomology of the resulting complex of sheaves as the definition of Hochschild cohomology of $D^{b}(X)$. This is the approach taken in Ref. 40 for Hochschild homology; a similar definition of Hochschild cohomology is adopted in Ref. 13. Another possibility is to consider the diagonal subvariety $\Delta \subset X \times X$ and define the Hochschild cohomology of $X$ to be the endomorphism algebra of $\Delta$ regarded as an object of $D^{b}(X \times X)$ 38. Remarkably, all these definitions give the same result for quasi-projective schemes 38. M. Kontsevich interpreted the last definition of $\operatorname{HH}^{*}(X)$ as computing the space of infinitesimal deformations of the bounded derived category of coherent sheaves on $X$ in the class of $A_{\infty}$ categories [25].

The special thing about the diagonal in $X \times X$ is that it is a geometric object representing the identity functor from the category of D-branes on $X$ to itself. That is, if one takes a D-brane on $X$, pulls it back to $X \times X$ using the projection to the first factor, tensors with the diagonal object, and then pushes down to $X$ using the projection to the second factor, then one gets back the same D-brane, up to isomorphism. Then endomorphisms of the diagonal D-brane parametrize infinitesimal deformations of the identity functor, i.e. infinitesimal deformations of the category of D-branes on $X$ 25]. Thus it is natural to define the Hochschild cohomology of the category of D-branes on $X$ as the endomorphism algebra of the diagonal D-brane on $X \times X$.

Using the "diagonal brane" definition, it is easy to see that for smooth quasi-projective $X$ the Hochschild cohomology is

$$
\mathrm{HH}^{n}(X)=\oplus_{p+q=n} H^{p}\left(\Lambda^{q} T X\right) .
$$

Indeed, if we are dealing with a B-brane on a manifold $Z$ which corresponds to the structure sheaf of a smooth submanifold $Y$, then its open string algebra is given by

$$
\oplus_{p, q} H^{p}\left(\Lambda^{q} N Y\right),
$$

where $N Y$ is the normal bundle of $Y$ in $Z$. The fermion number (i.e. grade) is $p+q$. More precisely, this bigraded vector space is the first term in a spectral sequence which converges to the space of physical open strings 23 . In our case $Z=X \times X$, and $Y=\Delta$, so $N Y=T X$, and the first term of the spectral sequence computing the space of physical open string states for $\Delta$ is precisely Eq. (5). The spectral sequence actually collapses in this case, so the Hochschild cohomology of $X$ is given by Eq. (15).

More generally, one notes that instead of the canonical resolution of the diagonal mentioned above one can take any other projective 
resolution. This is very useful, because the "canonical" resolution is inconvenient for computations: even for $A=\mathbb{C}[x]$, the algebra of polynomials in one variable, it has infinite length. In the next section we will be mostly interested in the case when $X$ is an affine space $V \simeq \mathbb{C}^{n}$. In this case a much more convenient free resolution of $\Delta$ is known, the Koszul resolution. To describe it, let $x_{1}, \ldots, x_{n}$ and $y_{1}, \ldots, y_{n}$ be affine coordinates on $V \times V$ and let us introduce $n$ fermionic variables $\theta_{1}, \ldots, \theta_{n}$. Consider the vector space $K$ of polynomial functions of all these variables. It is graded by the negative of the fermion number. It is easy to see that $K$ is a free graded module over $\mathbb{C}[V \times V]$ of rank $2^{n}$. Consider the following linear map on $K$ :

$$
k=\sum_{i=1}^{n}\left(x_{i}-y_{i}\right) \frac{\partial}{\partial \theta_{i}}
$$

Obviously, $k$ is a degree-one endomorphism of $K$ which squares to zero. The pair $(K, k)$ is the Koszul complex for $\Delta$. One can check that its cohomology is concentrated in degree zero and is isomorphic to $\mathbb{C}[V \times V] /(x-y) \simeq \mathbb{C}[V]$.

M. Kontsevich conjectured [25] that the "diagonal brane" construction will work in a similar way for the category of A-branes on a CalabiYau $X$. The category of A-branes is the derived category of the Fukaya category, which is an $A_{\infty}$ category whose objects are, roughly speaking, Lagrangian submanifolds with flat vector bundles. The diagonal brane is the diagonal submanifold in $X \times X$, where the second copy of $X$ is taken with a symplectic structure opposite to that on the first copy. $^{2}$ We will denote the second copy of $X$ with a reversed symplectic structure by $X^{o p}$. The vector bundle on $\Delta \subset X \times X^{o p}$ is taken to be the trivial rank one bundle. Kontsevich conjectured that the endomorphism algebra of this diagonal brane, regarded as an object of the derived Fukaya category, is isomorphic to the quantum cohomology ring of $X$ (which is the algebra of physical closed string states in the A-model on $X$ ). This conjecture remains unproved.

One can give a general (although somewhat nonrigorous) argument that the Hochschild cohomology of the category of D-branes defined as the endomorphism algebra of the diagonal brane is the space of physical

\footnotetext{
${ }^{2}$ Apparently, reversing the sign of the symplectic form is analogous to passing from $A$ to $A^{o p}$ for B-branes. This analogy would have a natural explanation if the Fukaya category were somehow related to the deformation quantization of $(X, \omega)$ : changing the sign of $\omega$ has the effect of replacing the quantized algebra of functions on $X$ with its opposite. A relation between the Fukaya category and deformation quantization has been conjectured by many people, including one of the authors of the present paper; unfortunately, the nature of such a relation remains elusive.
} 
closed string states. Consider a closed-string spherical worldsheet $\Sigma$ on which there lives some topological field theory $\mathcal{T}$. We can reinterpret it as an open-string disk worldsheet as follows. Let us draw an equatorial circle (seam) on $\Sigma$ and identify the upper and lower hemispheres by means of an antiholomorphic map. Let $\mathcal{T}^{o p}$ be the image of $\mathcal{T}$ under this map. (For B-model on a Calabi-Yau, $\mathcal{T}^{o p}$ is isomorphic to $\mathcal{T}$; for a Landau-Ginzburg model, $\mathcal{T}^{o p}$ differs from $\mathcal{T}$ only by the reversal of the sign of the superpotential; for A-model on a Calabi-Yau, $\mathcal{T}^{o p}$ differs from $\mathcal{T}$ by the reversal of the sign of the symplectic structure.) Then $\mathcal{T}$ living on $\Sigma$ is equivalent to $\mathcal{T} \otimes \mathcal{T}^{o p}$ living on the upper hemisphere, with some gluing condition on the boundary. This gluing condition corresponds to a D-brane on $X \times X^{o p}$, and it is chosen so that after reinterpreting a $\mathcal{T} \otimes \mathcal{T}^{o p}$ field configuration on the upper hemisphere as a $\mathcal{T}$ field configuration on the union of upper and lower hemispheres, the fields join smoothly on the boundary. This is a physical definition of the diagonal brane. If a single closed-string operator is inserted on $\Sigma$, we may choose the seam to pass through the insertion point, and then we should be able to interpret it as an open-string operator in $\mathcal{T} \otimes \mathcal{T}^{o p}$ inserted on the boundary of the disk. Continuing this line of argument, we see that the algebra of physical closed-string states should be isomorphic to the endomorphism algebra of the diagonal brane, and that this isomorphism should identify the closed-string topological metric with the open-string topological metric. ${ }^{3}$ In this paper we check these statements in the case of topological Landau-Ginzburg models on the affine space and on certain orbifolds of the affine space.

\section{LANDAU-GinZBURG MODELS ON AFFine SPACE}

Consider a topological Landau-Ginzburg model on the affine space $V \simeq \mathbb{C}^{n}$ with a polynomial superpotential $W: V \rightarrow \mathbb{C}^{4}$ To this data one can associate a CDG-algebra, i.e. a $\mathbb{Z}_{2}$-graded algebra with an odd derivation $Q: A \rightarrow A$ and an even element $B \in A$ such that $Q^{2} a=[B, a]$ for any $a \in A$. The derivation $Q$ is sometimes called a twisted differential (ordinary differentials satisfy $Q^{2}=0$ ). The notion of a CDG-algebra is a slight generalization of the notion of a $\left(\mathbb{Z}_{2^{-}}\right.$ graded) DG-algebra. In the Landau-Ginzburg case, $A=\mathbb{C}[V]$ (the algebra of polynomials on $V), Q=0$, and $B=W$. A CDG-module

\footnotetext{
${ }^{3}$ Recall that for any algebra or DG-algebra $A$ its Hochschild cohomology has the structure of a supercommutative algebra [12. This is in agreement with the fact that the algebra of physical closed-string states is always supercommutative.

${ }^{4}$ In what follows we will always assume that the critical set of $W$ is compact; this is necessary for the Landau-Ginzburg field theory to have a normalizable vacuum state.
} 
over a CDG-algebra $(A, Q, B)$ is a pair $(M, D)$, where $M$ is a graded module over $A$ and $D$ is an odd derivation of $M$ such that $D^{2} m=B m$ for any element $m \in M$. In the Landau-Ginzburg case, $Q=0$, so $D$ is simply an odd endomorphism of $M$ satisfying $D^{2}=W$.

As explained in Refs. [21, 22, the category of D-branes for the topological LG model is equivalent to a DG-category whose objects are free CDG-modules over the Landau-Ginzburg CDG-algebra, and morphisms are morphisms between CDG modules. That is, the space of morphisms between $(M, D)$ and $\left(M^{\prime}, D^{\prime}\right)$ is a differential graded vector space, which coincides with the space of morphisms between graded modules $M$ and $M^{\prime}$, as far as graded vector structure is concerned, while the differential is

$$
D_{M M^{\prime}}(\phi)=D^{\prime} \circ \phi-(-1)^{|\phi|} \phi \circ D, \quad \phi \in \operatorname{Hom}_{A}\left(M, M^{\prime}\right) .
$$

One can make an ordinary category out of this DG-category by redefining morphisms between $M$ and $M^{\prime}$ to be the cohomology of $D_{M M^{\prime}}$. This is the homotopy category of CDG-modules. In the affine case, this is the same as the derived category of CDG-modules. The space of physical open-string states is identified with the cohomology of $D_{M M^{\prime}}$. In particular, if we take $M=M^{\prime}$, then the algebra of physical openstring states is identified with the endomorphism algebra of $(M, D)$ in the derived category of CDG-modules.

If $M$ is a free $\mathbb{Z}_{2}$-graded module over $A=\mathbb{C}[V]$, then the endomorphism algebra of $M$ (in the category of graded $A$-modules) is simply the algebra of matrices with entries in $\mathbb{C}[V]$. This algebra is graded in an obvious way: endomorphisms which preserve the parity of elements of $M$ are considered even, while endomorphisms which flip the parity are considered odd. If we order the generators of $M$ so that even generators go first, then elements of $\operatorname{End}_{A}(M)$ can be written as $2 \times 2$ block matrices with entries in $A$, where the diagonal blocks are even, and the off-diagonal ones are odd. Such matrices are usually called supermatrices (over $A=\mathbb{C}[V]$ ). The problem of finding solutions to the equation $D^{2}=W$ can be thought of as the problem of factorizing $W$ into a product of two identical odd supermatrices with polynomial entries. We will sometimes refer to it as the matrix factorization problem.

The goal of this section is to compute the Hochschild cohomology of the category of D-branes in topological LG models and to compare it with the algebra of closed string states, which is known to be the so-called Jacobi ring of $W$, i.e.

$$
\mathrm{J}_{W}=\mathbb{C}[V] / I_{W}, \quad I_{W}=\left(\partial_{1} W, \ldots, \partial_{n} W\right)
$$


But first we need to give a definition of the Hochschild cohomology of a CDG-algebra. By analogy with the previous section, we would like to define it as the endomorphism algebra of the "diagonal" brane on $V \times V$, or more algebraically, as the endomorphism algebra of a $A=\mathbb{C}[V]$ regarded as an object of the derived category of CDG-bimodules over itself. That is, we would like to regard $A$ as a CDG-module over the tensor product of $(A, 0, W)$ with its opposite. Since $A$ is commutative, one may ask why we are retaining the word "opposite" here. We claim that in the world of CDG algebras taking an opposite of an algebra includes changing the sign of the special element $B$. Indeed, suppose we take some arbitrary $\mathrm{CDG}$-algebra $(A, Q, B)$. We cannot regard it as a CDG-module over itself by simply setting $D=Q$, because the defining relation for a CDG-module is $D^{2}(a)=B a$, while $Q$ satisfies $Q^{2} a=[B, a]$. Next, if we consider the tensor product of $(A, Q, B)$ with $\left(A^{o p}, Q, B\right)$, we still cannot regard $(A, Q, B)$ as a CDG-module over this tensor product algebra, because setting $D=Q$ still gives $D^{2}(a)=[B, a]$, while the defining relation of the CDG-module gives in this case

$$
D^{2}(a)=B a+a B
$$

However, if we take the tensor product of $(A, Q, B)$ with $\left(A^{o p}, Q,-B\right)$, then setting $D=Q$ does make $(A, Q)$ into a CDG-module over the tensor product algebra.

In the Landau-Ginzburg case, this means that we define Hochschild cohomology as the endomorphism algebra of $(\mathbb{C}[V], 0, W)$ regarded as a B-brane on $V \times V$ with the superpotential $W(x)-W(y)$, where $x, y$ are affine coordinates on the two copies of $V$. To compute this endomorphism algebra, we have to replace $(\mathbb{C}[V], 0, W)$ by a free CDGmodule which is isomorphic to $(\mathbb{C}[V], 0, W)$ in the sense of the derived category of CDG-modules.

We can simply guess the equivalent free CDG-module (a more rigorous justification is given in the end of this section). If $W$ were zero, then we would have to replace the structure sheaf of the diagonal with its free resolution. Since we are dealing with CDG-modules, we have to find instead a "twisted" resolution, i.e. a sequence of free $\mathbb{C}[V \times V]$-modules and morphisms between them such that the composition of successive morphisms is $W$ instead of zero. Therefore, we will do the following. We will take the Koszul resolution of the diagonal $\Delta \subset V \times V$, fold it modulo 2 (since our algebra is $\mathbb{Z}_{2}$-graded, complexes must also be $\mathbb{Z}_{2}$-graded), and then deform in a natural way the differential into a twisted differential. This will give us a free CDG-module on $V \times V$, 
and we will take its endomorphism algebra as the definition of the Hochschild cohomology for the category of D-branes.

First let us see how this works for the one-variable case, where $V \sim$ $\mathbb{C}$, and $A=\mathbb{C}[x]$. We are considering the diagonal submanifold $\Delta$ in $\mathbb{C}^{2}$ given by the equation $x=y$. The Koszul resolution is very short:

$$
\mathbb{C}[x, y] \stackrel{x-y}{\longrightarrow} \mathbb{C}[x, y]
$$

The folded resolution can be regarded as a $\mathbb{Z}_{2}$-graded module of the form $\mathbb{C}[x, y] \oplus \mathbb{C}[x, y]$ and the differential

$$
D=\left(\begin{array}{cc}
0 & 0 \\
x-y & 0
\end{array}\right)
$$

This is an honest differential satisfying $D^{2}=0$. On the other hand, a twisted differential would satisfy $D^{2}=W(x)-W(y)$. The correct deformation is obvious:

$$
D=\left(\begin{array}{cc}
0 & \frac{W(x)-W(y)}{x-y} \\
x-y & 0
\end{array}\right)
$$

Note that since $W(x)$ and $W(y)$ are polynomials, $D$ has polynomial entries. This matrix factorization has been previously considered in a similar context in Ref. [24].

Above $D$ was written as an odd supermatrix. Equivalently, we can think about such supermatrices as differential operators on functions of odd variables. (This is the approach we took in the last section to write down the Koszul resolution). In the fermionic language the deformed $D$ looks as follows:

$$
D=(x-y) \frac{\partial}{\partial \theta}+\frac{W(x)-W(y)}{x-y} \theta .
$$

According to Ref. [21, 22, 5], the endomorphism algebra of this brane is the cohomology of the following linear operator on the space of supermatrices with polynomial entries:

$$
\mathcal{D}: \phi \rightarrow D \phi-(-1)^{|\phi|} \phi D
$$

where $\phi$ is a supermatrix. For the operator $D$ as above, it is easy to see that the cohomology is purely even and isomorphic to the quotient vector space

$$
\mathbb{C}[x, y] / I, \quad I=\left(x-y, \frac{W(x)-W(y)}{x-y}\right)
$$

Obviously, this vector space is isomorphic to $\mathrm{J}_{W}=\mathbb{C}[x] /\left(\partial_{x} W(x)\right)$, as claimed. In fact, it is easy to see that the isomorphism holds on the level of algebras, rather than on the level of vector spaces. 
Next consider the case when $V \simeq \mathbb{C}^{n}$. Let us introduce convenient condensed notations:

$$
\mathbf{x}=\left(x_{1}, \ldots, x_{n}\right), \quad \mathbf{y}_{[i]} \mathbf{x}=\left(y_{1}, \ldots, y_{i}, x_{i+1}, \ldots, x_{n}\right)
$$

and for a polynomial $P \in \mathbb{C}[\mathbf{x}]$ define

$$
\Delta_{i}(P)=\frac{P\left(\mathbf{y}_{[i-1]} \mathbf{x}\right)-P\left(\mathbf{y}_{[i]} \mathbf{x}\right)}{x_{i}-y_{i}} \in \mathbb{C}[\mathbf{x}, \mathbf{y}] .
$$

To write the correct free CDG-module (the twisted resolution of the diagonal), we first write $W(\mathbf{x})-W(\mathbf{y})$ as a sum of polynomials

$$
W(\mathbf{x})-W(\mathbf{y})=\sum_{i=1}^{n}\left(x_{i}-y_{i}\right) \Delta_{i}(W) .
$$

We want to write $W(\mathbf{x})-W(\mathbf{y})$ as $D^{2}$, where $D$ is a odd endomorphism of free $\mathbb{Z}_{2}$-graded modules. The key point is that for each term $\Delta_{i}(W)$ we can accomplish this by mimicking the one-variable case. That is, if we let

$$
D_{i}=\left(x_{i}-y_{i}\right) \frac{\partial}{\partial \theta_{i}}+\Delta_{i}(W) \theta_{i}
$$

then $D_{i}$ is a odd endomorphism of free graded $\mathbb{C}[x, y]$-modules which satisfies $D_{i}^{2}=\Delta_{i}(W)$. Therefore if we let

$$
D=\sum_{i=1}^{n} D_{i}
$$

we will get the desired $D^{2}=W(\mathbf{x})-W(\mathbf{y})$. Moreover, if we take the limit $W \rightarrow 0$, the differential $D$ will reduce to $k$, the Koszul differential from the previous section. The corresponding matrix factorization has been previously discussed in Ref. [24].

Since the differential is given by a sum, and we have computed the cohomology of $D_{i}$ already, the cohomology of $D$ is simply

$$
\mathbb{C}[V \times V] / I, \quad I=\left(x_{1}-y_{1}, \ldots, x_{n}-y_{n}, \Delta_{1}(W), \ldots, \Delta_{n}(W)\right),
$$

Obviously, this is isomorphic to Eq. (6).

We end this section by explaining why the D-brane Eq. (11) deserves to be called the diagonal brane. The general definition of the diagonal is that it represents the identity functor in the category of D-branes on $\left(\mathbb{C}^{n}, W\right)$. In the present case, this means the following. Let $(R, D)$ be the matrix factorization of $W(\mathbf{x})-W(\mathbf{y})$ corresponding to Eq. (11). Thus $R$ is a free graded $A \otimes A$-module, where $A \simeq \mathbb{C}[\mathbf{x}]$, and $D$ is an odd endomorphism of $R$ satisfying $D^{2}=W(\mathbf{x})-W(\mathbf{y})$. Let $(N, F)$ be an arbitrary matrix factorization of $W(\mathbf{y})$ representing some Dbrane on $\left(\mathbb{C}^{n}, W\right)$. Thus $N$ is a free graded $A$-module, and $F$ is an odd 
endomorphism of $N$ satisfying $F^{2}=W(\mathbf{y})$. Consider the graded tensor product $\left(R \otimes_{A} N, D+F\right)$. The module $R \otimes_{A} N$ is a free $A$-module, and $D+F$ satisfies

$$
(D+F)^{2}=D^{2}+F^{2}=(W(\mathbf{x})-W(\mathbf{y}))+W(\mathbf{y})=W(\mathbf{x}) .
$$

Thus the pair $\left(R \otimes_{A} N, D+F\right)$ is a D-brane on $\left(\mathbb{C}^{n}, W\right)$ (of infinite rank). By definition, $(R, D)$ is the diagonal iff $\left(R \otimes_{A} N, D+F\right)$ is isomorphic to the original finite-dimensional brane $(N, F)$, for any $(N, F)$ (in the homotopy category of CDG-modules). This statement was proved in Ref. 24] (Prop. 23 of that paper).

\section{LG ORBIFOLDS}

4.1. Minimal model I. Consider the LG model with target $\mathbb{C} / \mathbb{Z}_{n}$ :

$$
W_{0}=x^{n}, \quad x \sim \zeta x, \zeta=e^{\frac{2 \pi i}{n}}
$$

According to Ref. [20, the chiral ring is simply the invariant part of the Jacobi ring, which is $\mathbb{C}[x] / x^{n-1}$. In other words, there are no chiral primaries in the twisted sectors. The only invariant is actually the identity element. Let us check this using the diagonal B-brane approach.

We consider a matrix factorization of the potential

$$
W=x^{n}-y^{n} .
$$

$W$ is a function on $\mathbb{C}^{2}$ invariant under a $\mathbb{Z}_{n} \times \mathbb{Z}_{n}$ action

$$
x \rightarrow \zeta^{k} x, \quad y \rightarrow \zeta^{k+l} y, \quad k, l \in \mathbb{Z} / n .
$$

In other words, $x$ has weight $(1,0)$, while $y$ has weight $(1,1)$. The corresponding twisted differential $D$ is

$$
D_{0}(x, y)=\left(\begin{array}{cc}
0 & \prod_{i=1}^{n-1}\left(x-\zeta^{i} y\right) \\
x-y & 0
\end{array}\right)
$$

$D_{0}$ is regarded as an odd endomorphism of a free $\mathbb{Z}_{2}$-graded module over $\mathbb{C}[x, y]$ of rank two, which we write as $M_{0}=M_{0}^{+} \oplus M_{0}^{-}$, where $M_{0}^{+} \simeq \mathbb{C}[x, y]$ is the even component, and $M_{0}^{-} \simeq \mathbb{C}[x, y]$ is the odd component.

We need to define a $G=\mathbb{Z}_{n} \times \mathbb{Z}_{n}$ action on $M_{0}$ so that $M_{0}$ becomes an equivariant module over $\mathbb{C}[x, y]$, and the twisted differential is an equivariant endomorphism. A $\mathbb{Z}_{n}$ action on a free module, like $M_{0}^{+}$ or $M_{0}^{-}$, is completely specified once we specify the action on the unit element, i.e. its weight. For the first $\mathbb{Z}_{n}$, we choose the weights to be 0 for $M_{0}^{+}$and 1 for $M_{0}^{-}$. This is the only way to make $D_{0}$ equivariant, up 
to an overall shift of weights. For the second $\mathbb{Z}_{n}$, no choice of weights works, so we have to equivariantize $D_{0}$, i.e. to add "image branes":

$D_{l}(x, y)=D_{0}\left(x, \zeta^{l} y\right)=\left(\begin{array}{cc}0 & \prod_{i=0, i \neq l}^{n-1}\left(x-\zeta^{i} y\right) \\ x-\zeta^{l} y & 0\end{array}\right), \quad l=1, \ldots, n-1$.

$D_{l}$ acts on a module $M_{l}$ which is isomorphic to $M_{0}$ as a $\mathbb{Z}_{2} \times \mathbb{Z}_{n}$-graded module. We take the diagonal brane $M$ to be the direct sum of all these branes, with the twisted differential $D$ being the sum of all $D_{l}$, $l=0, \ldots, n-1$. $D$ is equivariant with respect to the second $\mathbb{Z}_{n}$ if we let $\mathbb{Z}_{n}$ act on the summands $M_{l}$ by cyclic permutations.

Now let us compute the endomorphism algebra of the diagonal brane $(M, D)$. First we compute the endomorphisms disregarding the $\mathbb{Z}_{n} \times \mathbb{Z}_{n}$ action, and then project onto the invariant part. Since $M$ is a direct sum, we can compute morphisms between the summands, and then sum them up. It is also convenient to consider separately the morphisms from a brane $\left(M_{l}, D_{l}\right)$ to itself, and to its mirror images $\left(M_{k}, D_{k}\right), k \neq l$. An easy computation shows that the endomorphisms of $\left(M_{l}, D_{l}\right)$ (i.e. the cohomology of $D_{l}$ in the adjoint representation) is purely even and is the Jacobi ring of $W_{0}$. The space of morphisms between $\left(M_{l}, D_{l}\right)$ and $\left(M_{k}, D_{k}\right)$ is purely odd and one-dimensional. A basis element of this odd vector space will be called $\theta_{l, k}$. Since the odd components of $M_{l}$ have weight 1 with respect to the first $\mathbb{Z}_{n}$, one can easily see that $\theta_{k, l}$ has weight 1 . Projecting with respect to the first $\mathbb{Z}_{n}$, we see that no morphisms between a brane and its image survive. As for endomorphisms of $\left(M_{l}, D_{l}\right)$, only the invariant part of the Jacobi ring (which consists only of the subspace spanned by the identity) survives. Thus we get the direct sum of $n$ copies of the trivial algebra. Finally, we project with respect to the second $\mathbb{Z}_{n}$. This $\mathbb{Z}_{n}$ cyclically permutes all $M_{l}$, and therefore identifies all the $n$ copies of the trivial algebra.

4.2. Minimal model II. This is a generalization of the previous subsection. We consider a LG model with target $\mathbb{C} / \mathbb{Z}_{n}$ and a superpotential

$$
W_{0}=x^{n m}, \quad n, m \in \mathbb{N} .
$$

The orbifold group acts by

$$
x \mapsto \zeta^{k} x, \quad k \in \mathbb{Z} / n .
$$

The analysis is very similar to the previous subsection. The untwisted sector is the invariant part of the Jacobi ring. There are still $n-1$ image branes, and morphisms between different images are odd and have weight 1 under the first $\mathbb{Z}_{n}$ action. Hence the twisted sector 
is killed by the first $\mathbb{Z}_{n}$ projection. This agrees with the results of Ref. [20].

4.3. Gepner model for a Calabi-Yau 0-fold. This is a LG orbifold with a target $\mathbb{C}^{2} / \mathbb{Z}_{2}$ and superpotential

$$
W_{0}=x_{1}^{2}+x_{2}^{2} \text {. }
$$

The $\mathbb{Z}_{2}$ action is diagonal (flips the sign of both variables). E. Witten's argument 44] shows that this is a Gepner model for the CY 0-fold given as a hypersurface $x_{1}^{2}+x_{2}^{2}=0$ in $\mathbb{P}^{1}$. The latter consists of two points, so the corresponding chiral ring is two-dimensional (the direct sum of two copies of the trivial algebra). Let us check this using the diagonal brane approach.

We consider the superpotential

$$
W=x_{1}^{2}-y_{1}^{2}+x_{2}^{2}-y_{2}^{2}=W_{1}\left(x_{1}, y_{1}\right)+W_{2}\left(x_{2}, y_{2}\right) .
$$

To construct the diagonal brane, we factorize separately $W_{1}$ and $W_{2}$ and then take the $\mathbb{Z}_{2}$-graded tensor product of the corresponding branes. If we represent $D_{1}$ and $D_{2}$ by fermionic differential operators, this simply means that we let $D=D_{1}+D_{2}$, where $D_{1}^{2}=W_{1}$ and $D_{2}^{2}=W_{2}$. Since $D_{1}$ and $D_{2}$ anticommute, this gives $D^{2}=W$.

The factorizations of $W_{1}$ and $W_{2}$ are taken as in the previous subsection. Then we have to equivariantize with respect to $\mathbb{Z}_{2} \times \mathbb{Z}_{2}$. As above, equivariance with respect to the first $\mathbb{Z}_{2}$ forces the odd component of $M$ to have weight 1 , if the even component has weight 0 . Hence odd endomorphisms of $M$ have weight one, while even endomorphisms have weight 0 . Equivariance with respect to the second $\mathbb{Z}_{2}$ requires adding a single "image brane" $\left(M^{\prime}, D^{\prime}\right)$, where $M^{\prime} \simeq M$, and $D^{\prime}$ is

$$
D^{\prime}\left(x_{1}, x_{2}, y_{1}, y_{2}\right)=D\left(x_{1}, x_{2},-y_{1},-y_{2}\right) .
$$

The diagonal brane is the direct sum of $(M, D)$ and its image $\left(M^{\prime}, D^{\prime}\right)$. Let us compute its endomorphism algebra and then project onto the $\mathbb{Z}_{2} \times \mathbb{Z}_{2}$-invariant part. First consider the endomorphisms of $M$ and $M^{\prime}$. Since both $M$ and $M^{\prime}$ have factorized form (they are graded tensor products of $M_{1}$ and $M_{2}$ ), we can compute the endomorphisms of $M_{1}$ and $M_{2}$ and then tensor them. From the previous subsection we known that the endomorphism algebras of $M_{1}$ and $M_{2}$ are trivial (spanned by the identity). Hence the endomorphism algebras of $M$ and $M^{\prime}$ are also trivial. They also survive the first $\mathbb{Z}_{2}$ projection, while the second $\mathbb{Z}_{2}$ projection identifies them.

Now consider morphisms from $M$ to $M^{\prime}$. Using the results of the previous subsection, we see that the space of morphisms is one-dimensional and spanned by $\theta_{0,1}^{(1)} \otimes \theta_{0,1}^{(2)}$, where $\theta_{0,1}^{(i)}$ spans the space of morphisms from 
$M_{i}$ to its image under the second $\mathbb{Z}_{2}$. Thus the space of morphisms from $M$ to $M^{\prime}$ is purely even and one-dimensional. Since both $\theta$ 's have weight 1 under the first $\mathbb{Z}_{2}$, their product has weight 0 and survives the first $\mathbb{Z}_{2}$ projection. Finally, the second $\mathbb{Z}_{2}$ projection identifies morphisms from $M$ to $M^{\prime}$ and from $M^{\prime}$ to $M$. Thus the twisted sector contributes a single even element to the chiral ring. It is easy to see that this element squares to identity. Thus the endomorphism algebra of the diagonal brane isomorphic to the group ring of $\mathbb{Z}_{2}$. This is the desired result.

4.4. Gepner model for a Calabi-Yau $n-2$-fold. We consider the superpotential $W=x_{1}^{n}+\ldots+x_{n}^{n}$ on $\mathbb{C}^{n}$ with a diagonal $\mathbb{Z}_{n}$ action:

$$
x_{i} \mapsto \zeta x_{i} .
$$

This is a Gepner model for the Fermat hypersurface $x_{1}^{n}+\ldots+x_{n}^{n}=0$ in $\mathbb{P}^{n-1}$. Let us compute the endomorphisms of the diagonal brane. The computation proceeds along the same lines as in the previous subsection. We now have $n-1$ image branes (with respect to the second $\mathbb{Z}_{n}$ ). The untwisted sector gives us the invariant part of the Jacobi ring. Morphisms between a brane and its image have the form

$$
\otimes_{i=0}^{n-1} \theta_{k, l}^{(i)}
$$

Each $\theta$ is odd and has weight 1 under the first $\mathbb{Z}_{n}$, so the product has weight 0 and survives the first $\mathbb{Z}_{n}$ projection. It is even or odd depending on whether $n$ is even or odd. The second $\mathbb{Z}_{n}$ projection identifies all the twisted sector states with the same value of $k-l$. Hence the twisted sector contributes $n-1$ states to the chiral ring (labeled by $k-l \bmod n$ ).

Let us compare this with the B-model of the Fermat hypersurfaces. The case $n=2$ has been discussed in the previous subsection. For $n=3$ the Fermat hypersurface is a cubic curve in $\mathbb{P}^{2}$, i.e. an elliptic curve. Its $\mathrm{B}$-model is isomorphic to the exterior algebra with two odd generators, i.e. it has two-dimensional even subspace and two-dimensional odd subspace. On the LG side, the untwisted sector is spanned by $1, x_{1} x_{2} x_{3}$ (the invariant part of the Jacobi ring), while the twisted sector is odd and two-dimensional. Thus the two agree, as $\mathbb{Z}_{2}$-graded vector spaces. One can check that the ring structure also agrees.

For $n=4$ the Fermat hypersurface is a quartic in $\mathbb{P}^{3}$, i.e. a K3 surface. The B-model is a purely even algebra of dimension 24 . On the other hand, the $\mathbb{Z}_{4}$-invariant part of the Jacobi ring is easily seen to have dimension 21. The twisted sector gives the other 3 states. 
For $n=5$ we are dealing with a quintic in $\mathbb{P}^{4}$. The B-model has both an even and odd components. The even component has dimension 204. The odd component has dimension 4. It is easy to show that the invariant part of the Jacobi ring is indeed 204-dimensional. The odd component comes entirely from the twisted sector.

\section{Open-Closed CORRElators FOR some LANDAU-GinzBurG MODELS}

5.1. Closed topological metric from the open one. In this subsection we discuss how to deduce the 3-point topological closed-string correlators (the Yukawa couplings) in the diagonal brane approach and show that the result agrees with the conventional approach [39].

The closed strings form a supercommutative Frobenius algebra $A_{\mathrm{cl}}$ : there is a trace map $A_{\mathrm{cl}} \stackrel{\operatorname{tr}_{\mathrm{F}}}{\longrightarrow} \mathbb{C}$, which defines the scalar product on $A_{\mathrm{cl}}$ and determines the 3 -point correlator of closed string operators

$$
(a, b)=\operatorname{tr}_{\mathrm{F}}(a b), \quad\langle a b c\rangle=\operatorname{tr}_{\mathrm{F}}(a b c) .
$$

The algebra of open-string states $A_{\mathrm{op}}$ has the $A_{\mathrm{cl}}$-linear 'boundary

trace' map $A_{\mathrm{op}} \stackrel{\operatorname{tr}_{\mathrm{b}}}{\longrightarrow} A_{\mathrm{cl}}$, which expresses the boundary state corresponding to a boundary with an open string operator insertion. The composition of the boundary and Frobenius traces $\operatorname{Tr}=\operatorname{tr}_{\mathrm{F}} \operatorname{tr}_{\mathrm{b}}$ determines the scalar product and the 3-point correlator for open strings:

$$
(A, B)=\operatorname{tr}_{\mathrm{b}}(A B), \quad\langle A B C\rangle=\operatorname{tr}_{\mathrm{b}}(A B C) .
$$

The conjectured correspondence between the diagonal D-brane states and the closed string states implies that the Frobenius trace on $A_{\mathrm{cl}}$ should be equal to the combined trace $\operatorname{Tr}$ on the diagonal D-brane states. This would also imply the equality between their scalar products and 3-point correlators.

We will verify the equivalence of Frobenius and combined traces for a topological LG model with the superpotential $W(\mathbf{x}) \in \mathbb{C}[\mathbf{x}]$. In this case the algebra of closed strings is the Jacobi algebra $\mathrm{J}_{W}$ of Eq. (6) and the Frobenius trace $\operatorname{tr}_{\mathbf{x}}$ was determined by C. Vafa in Ref. [39]:

$$
\operatorname{tr}_{\mathbf{x}}(p)=\frac{1}{(2 \pi i)^{n}} \oint \frac{p d x_{1} \wedge \ldots \wedge d x_{n}}{\partial_{1} W \ldots \partial_{n} W}, \quad p \in \mathbb{C}[\mathbf{x}] .
$$

The integrand in this formula is a meromorphic $n$-form, and the integration goes over a Lagrangian $n$-cycle defined by the equations

$$
\left|\partial_{i} W\right|=\epsilon_{i}>0, \quad i=1, \ldots, n .
$$


The boundary trace formula for a B-brane in a LG model, presented by a matrix factorization, was derived in Ref. [22] (see also Ref. [17]):

$$
\operatorname{tr}_{\mathrm{b}}(O)=(1 / n !) \mathrm{S} \operatorname{Tr}\left((\partial D)^{\wedge n} O\right)
$$

where $O$ is an endomorphism regarded as a supermatrix with polynomial entries, STr is the supertrace, and

$$
(\partial D)^{\wedge n}=\sum_{\sigma \in S_{n}}(-1)^{\operatorname{sign}(\sigma)} \partial_{\sigma(1)} D \cdots \partial_{\sigma(n)} D,
$$

where $S_{n}$ is the symmetric group of $n$ elements.

Since the space of endomorphisms of the diagonal B-brane is isomorphic (up to the BRST equivalence) to the Jacobi ring $\mathrm{J}_{W(\mathbf{x})}$, to prove the equivalence between closed- and open-string traces it is sufficient to show that

$$
\operatorname{tr}_{\mathrm{F}} \operatorname{tr}_{\mathrm{b}}(f(\mathbf{x}) \operatorname{Id})=\operatorname{tr}_{\mathbf{x}}(f)
$$

Here $f(\mathbf{x})$ is any polynomial, and Id is the identity endomorphism of the diagonal B-brane.

The relation (17) has an interesting algebraic corollary. Let $A^{*}$ be the dual space of an algebra $A$, then the multiplication map $A \stackrel{m}{\longrightarrow} A \otimes A$ has a dual map $A^{*} \otimes A^{*} \stackrel{\Delta}{\rightarrow} A^{*}$, which is called comultiplication. The scalar product (12) establishes a canonical isomorphism between the Frobenius algebra $A_{\mathrm{cl}}=\mathbb{C}[\mathbf{x}] / I_{W}$ and its dual, so there is a comultiplication map

$$
A_{\mathrm{cl}} \stackrel{\Delta}{\rightarrow} A_{\mathrm{cl}} \otimes A_{\mathrm{cl}}
$$

with the property

$$
\Delta(a)=(a \otimes 1) \Delta(1)=(1 \otimes a) \Delta(1) .
$$

The relation (17) implies that

$$
\operatorname{tr}_{\mathrm{b}}(\mathrm{Id})=\Delta(1) \in\left(\mathbb{C}[\mathbf{x}] / I_{W}\right) \otimes\left(\mathbb{C}[\mathbf{y}] / I_{W}\right)
$$

As the first step of proving the relation (17), we are going to derive a convenient formula for $\operatorname{tr}_{b}(\mathrm{Id})$ :

$$
\operatorname{tr}_{\mathrm{b}}(\mathrm{Id})=\frac{1}{(2 n) !} \mathrm{S} \operatorname{Tr}(\partial D)^{\wedge 2 n}=\operatorname{det}\left\|\Delta_{i}\left(\partial_{j} W\right)\right\|_{i, j=1}^{n}
$$

where $\partial_{j} W(\mathbf{x})=\partial W(\mathbf{x}) / \partial x_{j}$ and $\Delta_{i}$ is defined by Eq. (8). Indeed, if we expand the $2 n$-th power of the sum (10), then the supertrace of an 
individual monomial is zero, unless it contains all $\theta$ 's and all $\partial / \partial \theta$ 's. Therefore, if we lump all variables together $\mathbf{z}=(\mathbf{x}, \mathbf{y})$, then

$$
\mathrm{S} \operatorname{Tr}(\partial D)^{\wedge 2 n}=\frac{(2 n) !}{2^{n}} \sum_{\sigma \in S_{2 n}}(-1)^{\operatorname{sign}(\sigma)} \prod_{i=1}^{n} C_{i}\left[z_{\sigma(2 i)}, z_{\sigma(2 i+1)}\right]
$$

where

$$
C_{i}\left[z_{j}, z_{k}\right]=\operatorname{STr}_{i}\left(\frac{\partial D_{i}}{\partial z_{j}} \frac{\partial D_{i}}{\partial z_{k}}\right)
$$

while $D_{i}$ is defined by Eq. (10) and $\mathrm{STr}_{i}$ denotes the trace over the space $\left(1, \theta_{i}\right)$. A direct computation shows that if $\left\{z_{j}, z_{k}\right\} \cap\left\{x_{i}, y_{i}\right\}=\emptyset$, then $C_{i}\left[z_{j}, z_{k}\right]=0$ and the only non-zero factors are

$$
\begin{aligned}
C_{i}\left[x_{j}, y_{i}\right] & =-C_{i}\left[y_{i}, x_{j}\right]=\Delta_{i}\left(\partial_{j} W\right), & j \leq i, \\
C_{i}\left[x_{i}, y_{j}\right] & =-C_{i}\left[y_{j}, x_{i}\right]=\Delta_{i}\left(\partial_{j} W\right), & j \geq i .
\end{aligned}
$$

Thus, a non-zero supertrace $\mathrm{STr}_{i}$ must contain at least one derivative over an $i$-th variable $(x$ or $y$ ). The distributions of derivatives over the supertraces, which do not produce zero factors, can be enumerated by the elements $\sigma \in S_{n}$ : if $\sigma(i)=i$, then the supertrace (23) includes the derivatives over $x_{i}$ and $y_{i}$; if $\sigma(i) \neq i$, then one derivative of (23) should be over the $\sigma(i)$-th element of the list $\mathbf{y}_{[i]} \mathbf{x}$ and the other should be either over $x_{i}$ or over $y_{i}$ depending on whether $\sigma^{-1}(i)>i$ or $\sigma^{-1}(i)<$ $i$. This combinatorics together with the expressions (24) leads to the formula (21).

It remains to apply the Frobenius trace to the r.h.s. of Eq. (21) multiplied by $f(\mathbf{x})$. To do this, it is convenient to assume that all critical points of $W$ are non-degenerate. Since the set of such superpotentials is an open and dense subset of all admissible superpotentials, and the l.h.s. of Eq. (17) is a continuous function of the coefficients of $W$, it is sufficient to prove Eq. (17) in this special case. For $W$ with only non-degenerate critical points, the trace function Eq. (14) becomes

$$
\operatorname{tr}_{\mathrm{F}}(p)=\sum_{\text {crit } \mathrm{W}} \frac{p(\mathbf{x})}{\operatorname{Hess} W(\mathbf{x})}
$$

where Hess $W$ denotes the Hessian of $W$, and the sum is over all critical points of $W$. Thus we simply have to evaluate the r.h.s. of Eq. (21) at the critical points of $W(\mathbf{x})-W(\mathbf{y})$, which are pairs of critical points of $W(\mathbf{x})$. 
Let $\mathbf{x}$ and $\mathbf{y}$ be two critical points of $W$. If $\mathbf{x} \neq \mathbf{y}$, then $\operatorname{tr}_{\mathrm{b}}(\mathrm{Id})=0$ in view of Eq. $(21)^{5}$. Indeed, let

$$
r_{i}=\left(\Delta_{i}\left(\partial_{j} W\right)\right)_{j=1}^{n}
$$

be the rows of the matrix of Eq. (21). Obvious cancellations lead to the following identity:

$$
\sum_{i=1}^{n}\left(x_{i}-y_{i}\right) r_{i}=\left(\partial_{j} W(\mathbf{x})-\partial_{j} W(\mathbf{y})\right)_{j=1}^{n} .
$$

Since $\mathbf{x}$ and $\mathbf{y}$ are critical points of $W$, the r.h.s. of this equation is zero, and if $\mathbf{x} \neq \mathbf{y}$, then this means that the rows $r_{i}$ are linearly dependent, and the determinant in Eq. (21) is equal to zero.

Thus, only the critical points on the diagonal, i.e. the ones for which $\mathbf{x}=\mathbf{y}$, contribute to the sum (26). Expanding $W$ into a Taylor series to quadratic order, one can easily see that the r.h.s. of Eq. (21) evaluated on such a critical point is equal to Hess $W$. Hence we get:

$$
\operatorname{tr}_{\mathrm{F}} \operatorname{tr}_{\mathrm{b}}(f(\mathbf{x}) \mathrm{Id})=\sum_{\text {crit } \mathrm{W}} \frac{f(\mathbf{x})}{\operatorname{Hess} W(\mathbf{x})}=\operatorname{tr}_{\mathbf{x}}(f) .
$$

This completes the proof of Eq. (17).

As another example, let us deduce the topological closed-string metric for the elliptic curve at the Fermat point, using the LG representation and the diagonal brane approach. First, let us state the expected answer. The closed-string algebra of an elliptic curve is isomorphic to the exterior algebra with two generators $\xi_{1}, \xi_{2}$. Thus the space of physical closed-string states is four-dimensional, with two-dimensional even subspace (the sum of $H^{0}\left(\varnothing_{X}\right)$ and $H^{1}\left(T_{X}\right)$ ) and two-dimensional odd subspace (the sum of $H^{0}\left(T_{X}\right)$ and $H^{1}\left(\varnothing_{X}\right)$ ). The trace on this algebra is nonvanishing only on the one-dimensional subspace spanned by $\xi_{1} \xi_{2}$, which geometrically corresponds to $H^{1}\left(T_{X}\right)$.

In the LG approach, the states corresponding to $\xi_{1}$ and $\xi_{2}$ come from the twisted sector, while 1 and $\xi_{1} \xi_{2}$ span the $\mathbb{Z}_{3}$-invariant part of the Jacobi ring of the superpotential

$$
W=x_{1}^{3}+x_{2}^{3}+x_{3}^{3} .
$$

If we think about the closed-string space of states as the endomorphism algebra of a diagonal brane on $\left(\mathbb{C}^{3} / \mathbb{Z}_{3}\right) \times\left(\mathbb{C}^{3} / \mathbb{Z}_{3}\right)$, then the endomorphisms corresponding to $\xi_{1}$ and $\xi_{2}$ are off-diagonal (they correspond to strings from a brane to one of its images under a $\left.\mathbb{Z}_{3}\right)$, and it follows from Eq. (15) that their trace vanishes. As for the endomorphisms

\footnotetext{
${ }^{5}$ We are thankful to Christiaan Hofman for pointing out an error in our original proof and for suggesting the correct one.
} 
corresponding to 1 and $\xi_{1} \xi_{2}$, they are insensitive to the orbifolding and their properties are exactly the same as in the LG model on the affine space. We already know that the open-string trace for these endomorphisms coincides with the closed-string trace Eq. (14), provided we correctly identify endomorphisms with the elements of the Jacobi ring. The endomorphism 1 corresponds to the identity in the Jacobi ring and its trace is zero, according to Eq. (14). The endomorphism $\xi_{1} \xi_{2}$ (which geometrically represents a basis element for $H^{1}(T)$ ) corresponds to the element $x_{1} x_{2} x_{3}$ in the Jacobi ring, and its trace is a nonzero constant, according to Eq. (14). Thus we have recovered the correct Yukawa couplings for the elliptic curve by regarding the closed-string algebra as the Hochschild cohomology of the category of D-branes for the corresponding LG orbifold.

5.2. Bulk-boundary maps. Let us consider the simplest open-closed correlator: 2-point function on a disk, with one boundary and one bulk insertion. It can be regarded as a linear function

$$
A_{\mathrm{op}} \otimes A_{\mathrm{cl}} \rightarrow \mathbb{C} \text {. }
$$

Using the topological metric on $V_{o}$, it can also be regarded as a map

$$
\iota: A_{\mathrm{cl}} \rightarrow A_{\mathrm{op}}
$$

This is known as the bulk-boundary map; it is the dual of the boundary trace $\operatorname{tr}_{\mathrm{b}}$. Of course, for every choice of a D-brane, we get a $A_{\mathrm{op}}$, and therefore there are as many maps $\iota$ as there are D-branes. Axioms of Topological Field Theory with boundaries require $\iota$ to be an algebra homomorphism. In this subsection we will demonstrate that the bulkboundary map comes for free if we identify $A_{\mathrm{cl}}$ with the Hochschild cohomology of the category of topological D-branes. We will also check in some examples that the resulting bulk-boundary map is the correct one, i.e. it coincides with the one derived by physical methods.

Let $\mathcal{A}=(A, Q, B)$ be a CDG algebra, and suppose the category of D-branes is the derived category of CDG modules over $\mathcal{A}$. (The case of DG algebras and modules is a special case of this, corresponding to $B=0$.) Consider $^{6}$ the Hochschild cohomology of $\mathcal{A}$, defined as

$$
\mathrm{HH}^{*}(\mathcal{A})=\operatorname{Ext}_{\mathcal{A} \otimes \mathcal{A}^{o p}}^{*}(\mathcal{A}, \mathcal{A}) .
$$

That is, it is the endomorphism algebra of $\mathcal{A}$ regarded as an object of the derived category of modules over $\mathcal{A} \otimes \mathcal{A}^{o p}$. Let $\mathcal{M}$ be some object of the derived category of modules over $\mathcal{A}$, and let $\phi$ be an endomorphism

\footnotetext{
${ }^{6}$ The following construction was explained to us by D. Orlov.
} 
of $\mathcal{M}$. Then for any element $\alpha \in \operatorname{HH}^{*}(\mathcal{A})$ we may consider $\alpha \otimes \phi$, which is an endomorphism of

$$
\mathcal{A} \otimes_{\mathcal{A}} \mathcal{M} \in D^{b}(\mathcal{A})
$$

But the latter object is isomorphic to $\mathcal{M}$. Hence we may regard $\alpha \otimes \phi$ as as endomorphism of $\mathcal{M}$. Thus we get a linear map

$$
\kappa_{\mathcal{M}}: \mathrm{HH}^{*}(\mathcal{A}) \otimes \operatorname{End}(\mathcal{M}) \rightarrow \operatorname{End}(\mathcal{M})
$$

Obviously, this makes $\operatorname{End}(\mathcal{M})$ a (left) graded module over $\operatorname{HH}^{*}(\mathcal{A})$. Similarly, one can define the right $\operatorname{HH}^{*}(\mathcal{A})$-module structure on $\operatorname{End}(\mathcal{M})$. More generally, if we consider any two objects $\mathcal{M}$ and $\mathcal{N}$, the space of morphisms between them has a natural structure of a graded bimodule over $\mathrm{HH}^{*}(\mathcal{A})$. We define the bulk-boundary map $\iota_{\mathcal{M}}$ to be

$$
\iota_{\mathcal{M}}(\alpha)=\kappa_{\mathcal{M}}(\alpha \otimes 1) .
$$

It is trivial to see that this is an algebra homomorphism, as required.

Let us perform a simple check of this prescription for defining $\iota$. In the case of a LG model on an affine space $V$, the disk correlator has been computed in Ref. [22], and the corresponding bulk-boundary map is given by

$$
\iota_{\mathcal{M}}: f \mapsto f \mathrm{id}_{M}
$$

where $f$ is an element of the Jacobi ring $\mathrm{J}_{W}=\mathbb{C}[V] / \partial W$, and $\mathrm{id}_{M}$ is the identity endomorphism of a free CDG-module $\mathcal{M}=(M, D)$ over the CDG algebra $(\mathbb{C}[V], 0, W)$. It is implicit in this formula that $f$ is lifted to a polynomial on $V$. Although lifting an element of the Jacobi ring to an element of $\mathbb{C}[V]$ involves a choice, the above formula for $\iota_{\mathcal{M}}$ is well-defined. Indeed, if we replace $f$ by

$$
f+\sum_{i=1}^{n} g_{i} \partial_{i} W
$$

where $g_{i}$ are some polynomials on $V$, then $\iota_{\mathcal{M}}(f)$ will change by

$$
\left[D, \sum_{i} g_{i} \partial_{i} D\right] \text {, }
$$

which is homotopic to zero (or in other words, is BRST-trivial).

On the other hand, we established in Section 3 that the Jacobi ring is isomorphic to the endomorphism algebra of the "diagonal brane" on $V \times V$, the isomorphism being given by

$$
f \mapsto f \operatorname{id}_{R}
$$

where $R$ is the twisted Koszul resolution of the diagonal in $V \times V$. From this it follows that the $\operatorname{HH}^{*}(\mathcal{A})$-module structure on $\operatorname{End}(\mathcal{M})$ is very 
simple: it is induced by the $A$-module structure on $\operatorname{End}_{A}(M)$. Hence the abstract map $\iota_{\mathcal{M}}$ defined in Eq. (29) coincides with Eq. (30).

\section{Discussion}

In this paper we have shown that for topological Landau-Ginzburg models on the affine space (as well as on some quotients of the affine space) the algebra of physical closed-string states $^{7}$ is isomorphic to the Hochschild cohomology of the category of D-branes. The algebra structure on the Hochschild cohomology is given by the standard cup product. Moreover, using the open-string topological metric (in mathematical terms, the Serre functor on the category of D-branes), one can define a scalar product on the Hochschild cohomology, making it into a supercommutative Frobenius algebra. We showed that this scalar product agrees with the closed-string topological metric.

An important question is whether more complicated correlators can be deduced by studying the Hochschild cohomology of the category of D-branes. This is not completely trivial even at tree level (i.e. in genus zero). While two- and three-point correlators of gravitational primaries in genus zero are encoded in the Frobenius algebra structure, higherpoint correlators can be described by a germ of a Frobenius manifold (whose tangent space at the base point is the Frobenius algebra just mentioned.) In the case of the B-model of a Calabi-Yau manifold, the corresponding formal Frobenius manifold was constructed in Ref. [2]. Frobenius manifolds related to Landau-Ginzburg models are discussed in many papers, see e.g. Ref. 32]. It would be interesting to understand how to construct all these Frobenius manifolds in a uniform manner, starting from natural algebraic structures on the Hochschild complex of a category of topological D-branes. The key property needed for such a construction seems to be the existence of the open-string metric, i.e. a trivial Serre functor, on the category of D-branes.

A further question is how to reconstruct open-closed correlators. We only considered the simplest example (disk correlator with one boundary and one bulk insertion) and showed how to recover it from the $\mathrm{HH}^{*}(\mathcal{A})$-module structure on the space of endomorphisms of any Dbrane.

Although in this paper we have focused on topological string theories, it is tempting to conjecture that something similar holds for bosonic strings. A matter CFT with central charge $c=26$ coupled to diffeomorphism ghosts can be thought of as a topological field theory [9, 29, 10, 3, 33, 43, and can be coupled to topological gravity in

\footnotetext{
${ }^{7}$ We are talking about gravitational primaries only.
} 
the usual manner. This gives an alternative description of the bosonic string, which is similar to that of topological string theory. However, the physical spectrum of the bosonic string theory is related not to the BRST cohomology of the TFT, but to its semi-relative cohomology [46, 43, 15]. In particular, one cannot classify physical states as being gravitational primaries or descendants, in general, and it is not clear what the relation between physical closed string states and the Hochschild cohomology could be. A good example to look at is noncritical bosonic string theory with $c<1$. Some of the bosonic $c<1$ backgrounds are equivalent in a nontrivial way to topological string theories of the kind discussed in this paper [42, 9, 8, 29, and it would be interesting to rephrase our results in terms of the conventional formulation of the bosonic string.

\section{ACKNOWLEDGMENTS}

A.K. would like to thank Volodya Baranovsky, Ezra Getzler, Kentaro Hori, Dima Orlov, and Sasha Voronov for help at various stages. A.K. is also grateful to the Department of Mathematics of Northwestern University and the Erwin Schrödinger Institute for hospitality while this work was being completed. L. R. is very grateful to Mikhail Khovanov for numerous discussions of the category of matrix factorizations. This work was supported in part by the DOE grant DE-FG03-92-ER40701 and by the NSF grant DMS-0196131.

\section{REFERENCES}

[1] S. K. Ashok, E. Dell'Aquila and D. E. Diaconescu, Fractional branes in Landau-Ginzburg orbifolds, arXiv:hep-th/0401135.

[2] S. Barannikov and M. Kontsevich, Frobenius manifolds and formality of Lie algebra of polyvector fields, Internat. Math. Res. Notices no. 4 (1998) 201 arXiv:alg-geom/9710032.

[3] M. Bershadsky, W. Lerche, D. Nemeschansky and N. P. Warner, Extended $N=2$ superconformal structure of gravity and $W$ gravity coupled to matter, Nucl. Phys. B 401, 304 (1993) arXiv:hep-th/9211040.

[4] I. Brunner, M. R. Douglas, A. E. Lawrence and C. Romelsberger, D-branes on the quintic, JHEP 0008, 015 (2000) arXiv:hep-th/9906200.

[5] I. Brunner, M. Herbst, W. Lerche and B. Scheuner, Landau-Ginzburg realization of open string TFT, arXiv:hep-th/0305133

[6] I. Brunner and V. Schomerus, On superpotentials for D-branes in Gepner models, JHEP 0010, 016 (2000) arXiv:hep-th/0008194.

[7] D. E. Diaconescu and M. R. Douglas, D-branes on stringy Calabi-Yau manifolds, arXiv:hep-th/0006224.

[8] R. Dijkgraaf and E. Witten, Mean Field Theory, Topological Field Theory, And Multimatrix Models, Nucl. Phys. B 342, 486 (1990). 
[9] J. Distler, 2-D Quantum Gravity, Topological Field Theory And The Multicritical Matrix Models, Nucl. Phys. B 342, 523 (1990).

[10] B. Gato-Rivera and A. M. Semikhatov, Minimal models from $W$ constrained hierarchies via the Kontsevich-Miwa transform, Phys. Lett. B 288, 38 (1992) arXiv:hep-th/9204085.

[11] D. Gepner, Exactly Solvable String Compactifications On Manifolds Of SU(N) Holonomy, Phys. Lett. B 199, 380 (1987).

[12] M. Gerstenhaber, The cohomology structure of an associative ring, Ann. of Math. 2 (1963) 267.

[13] M. Gerstenhaber and S. D. Schack, Algebraic cohomology and deformation theory, in: Deformation theory of algebras and structures and applications (Il Ciocco, 1986), pp. 11-24, Kluwer, 1988.

[14] E. Getzler, Batalin-Vilkovisky Algebras And Two-Dimensional Topological Field Theories, Commun. Math. Phys. 159, 265 (1994) arXiv:hep-th/9212043.

[15] E. Getzler, Two-Dimensional Topological Gravity And Equivariant Cohomology, Commun. Math. Phys. 163, 473 (1994) arXiv:hep-th/9305013.

[16] M. Gutperle and Y. Satoh, D-branes in Gepner models and supersymmetry, Nucl. Phys. B 543, 73 (1999) arXiv:hep-th/9808080.

[17] M. Herbst and C. I. Lazaroiu, "Localization and traces in open-closed topological Landau-Ginzburg models," arXiv:hep-th/0404184.

[18] M. Herbst, C. I. Lazaroiu and W. Lerche, Superpotentials, $A_{\infty}$ relations and WDVV equations for open topological strings, arXiv:hep-th/0402110.

[19] K. Hori and J. Walcher, F-term equations near Gepner points, arXiv:hep-th/0404196

[20] K. A. Intriligator and C. Vafa, Landau-Ginzburg Orbifolds, Nucl. Phys. B 339, 95 (1990).

[21] A. Kapustin and Y. Li, D-branes in Landau-Ginzburg models and algebraic geometry, JHEP 0312, 005 (2003) arXiv:hep-th/0210296.

[22] A. Kapustin and Y. Li, Topological correlators in Landau-Ginzburg models with boundaries, arXiv:hep-th/0305136.

[23] S. Katz and E. Sharpe, D-branes, open string vertex operators, and Ext groups, arXiv:hep-th/0208104

[24] M. Khovanov and L. Rozansky, Matrix factorizations and link homology, arXiv:math.QA/0401268

[25] M. Kontsevich, Homological Algebra of Mirror Symmetry, arXiv:alg-geom $/ 9411018$

[26] T. Kugo, H. Kunitomo and K. Suehiro, Nonpolynomial Closed String Field Theory, Phys. Lett. B 226, 48 (1989).

[27] C. I. Lazaroiu, On the structure of open-closed topological field theory in two dimensions, Nucl. Phys. B 603, 497 (2001) arXiv:hep-th/0010269.

[28] C. I. Lazaroiu, On the boundary coupling of topological Landau-Ginzburg models, arXiv:hep-th/0312286.

[29] K. Li, Topological Gravity With Minimal Matter, Nucl. Phys. B 354, 711 (1991).

[30] B. H. Lian and G. J. Zuckerman, New perspectives on the BRST algebraic structure of string theory, Commun. Math. Phys. 154, 613 (1993) arXiv:hep-th/9211072. 
[31] B. H. Lian and G. J. Zuckerman, Some Classical And Quantum Algebras, arXiv:hep-th/9404010

[32] Yu. I. Manin, Three constructions of Frobenius manifolds: a comparative study, in: Sir Michael Atiyah, a great mathematician of the twentieth century, Asian J. of Math. 3 (1999) 179 arXiv:math.QA/9801006.

[33] S. Mukhi and C. Vafa, Two-dimensional black hole as a topological coset model of $c=1$ string theory, Nucl. Phys. B 407, 667 (1993) arXiv:hep-th/9301083.

[34] M. Penkava and A. Schwarz, $A_{\infty}$ Algebras And The Cohomology Of Moduli Spaces, arXiv:hep-th/9408064

[35] M. Penkava and A. Schwarz, On some algebraic structure arising in string theory, arXiv:hep-th/9212072.

[36] A. Recknagel and V. Schomerus, D-branes in Gepner models, Nucl. Phys. B 531, 185 (1998) arXiv:hep-th/9712186.

[37] M. Saadi and B. Zwiebach, Closed String Field Theory From Polyhedra, Annals Phys. 192, 213 (1989).

[38] R. G. Swan, Hochschild cohomology of quasiprojective schemes, J. Pure Appl. Algebra 110 (1996) 57.

[39] C. Vafa, Topological Landau-Ginzburg Models, Mod. Phys. Lett. A 6, 337 (1991).

[40] C. A. Weibel and S. C. Geller, Étale descent for Hochschild and cyclic homology, Comment. Math. Helv. 66 (1991) 368.

[41] E. Witten, Noncommutative Geometry And String Field Theory, Nucl. Phys. B 268, 253 (1986).

[42] E. Witten, On The Structure Of The Topological Phase Of Two-Dimensional Gravity, Nucl. Phys. B 340, 281 (1990).

[43] E. Witten, Chern-Simons gauge theory as a string theory, Prog. Math. 133, 637 (1995) arXiv:hep-th/9207094.

[44] E. Witten, Phases of $N=2$ theories in two dimensions, Nucl. Phys. B 403, 159 (1993) arXiv:hep-th/9301042.

[45] E. Witten and B. Zwiebach, Algebraic structures and differential geometry in $2 D$ string theory, Nucl. Phys. B 377, 55 (1992) arXiv:hep-th/9201056.

[46] B. Zwiebach, Closed string field theory: Quantum action and the B-V master equation, Nucl. Phys. B 390, 33 (1993) arXiv:hep-th/9206084.

California Institute of Technology, Department of Physics, Pasadena, CA 91125

E-mail address: kapustin@theory.caltech.edu

University of North Carolina, Department of Mathematics, Chapel

HiLL, NC 27599

E-mail address: rozansky@math.unc.edu 